\title{
MONOPOLISTIC COMPETITION IN A LARGE ECONOMY*
}

\author{
Tapan BISwAS
}

Abstract: For a small economy, the equilibrium under monopolistic competition may not be pareto optimal. The paper deals with the condition for the existence and pareto optimality of equilibrium under monopolistic competition in a large economy with differentiated products.

\section{INTRODUCTION}

In recent years, a large body of literature has emerged exploring the implications of monopolistic competition in a general equilibrium framework. The centre of research in this area is related to trade in differentiated products or intra-industry trade in open economies. The interested reader is referred to Dixit and Stiglitz (1977), Dixit and Norman (1980), Krugman (1979) and Helpman (1984). Monopolistic competition presumes the potential existence of a large number of firms. Therefore, it is appropriate to relate the discussion to a large economy, i.e., an economy where the number of individuals, involved in the production and the consumption of various goods, is large. There is also another reason for considering large economies in this context. In most of the wellknown works in this area, the marginal utility of income is assumed to be constant with respect to price changes (either explicitly or implicitly) for technical reasons. This is done by either restrictions imposed on the utility functions of the consumers (Dixit and Stiglitz (1977), Dixit and Norman (1980)) or by assuming that the variety of consumption goods available is very large (Krugman (1979)). The later approach has a problem. Since the variety of output produced is determined endogenously in the model, the assumption of a large variety of output may not be internally consistent with the model. However, one can show that as the number of agents in the economy becomes infinitely large, so does the variety of outputs produced. Therefore, it is desirable to consider an economy with large population in the context of monopolistic competition. We shall consider the model proposed by Krugman (1977), assuming constancy of the marginal utility of income (which, it is claimed, follows from his assumption of a large variety of outputs) rather than imposing stringent conditions on the utility function in such a way that the marginal utility of income remains invariant with respect to a change in any of the output prices.

* I am indebted to the referee of this journal for useful comments. The responsibility for any error lies entirely with me.

Originally published in Keio Economic Studies (1990), 26, 65-71. 
We begin by introducing the Krugman model (1979). Consider an economy with one scarce factor of production (labor) producing a range of outputs $x_{i}$, $i=1,2, \cdots, n$, where the index $i$ refers to the type of output. The production functions are identical across the variety of outputs produced. They are expressed in the following linear form and are apparently subject to increasing returns.

$$
L_{i}=\alpha+\beta x_{i} ; \quad \alpha>0, \quad \beta>0
$$

where $L_{i}$ is the amount of labor needed to produce $x_{i}$. Under the assumption of full employment,

$$
L=\sum_{i=1}^{n} L_{i} \quad \text { and } \quad x_{i}=L c_{i}
$$

The number of economic agents (workers and consumers) in the entire economy is denoted by $L$. Per capita consumption of the $i$-th variety of output is denoted by $c_{i}$. The total output of the $i$-th firm is denoted by $x_{i}$. It is assumed that all agents have identical concave utility functions of the following form,

$$
U=\sum_{i=1}^{n} v\left(c_{i}\right), \quad v(0)=0, \quad v^{\prime}>0, \quad v^{\prime \prime}<0
$$

We shall further assume that both $\lim _{c_{\mathrm{i}} \rightarrow 0} v^{\prime}\left(c_{i}\right)$ and $\lim _{c_{i} \rightarrow 0} v^{\prime \prime}\left(c_{i}\right) c_{i}$ exist (bounded in the limit). Because of symmetry in both production and consumption, the prices as well as the quantities for each commodity should be identical in equilibrium.

$$
p_{i}=p \quad \text { and } \quad x_{i}=x
$$

Krugman (1979) assumes that in equilibrium, the variety of output $(n)$ is large. Therefore, he treats the marginal utility of income $(\lambda)$ as a constant with respect to changes in prices. Intuitively, since the proportion of income spent on any particular commodity is very small, the effect of the change of any price on the marginal utility of income should be negligible. A formal proof of the result $\lim _{n \rightarrow \infty}\left(\delta \lambda / \delta p_{i}\right)=0$ is provided in the Appendix.

The validity of Krugman's assumption that $n$ is infinitely large, is open to question in a finite agent economy where $n$ is endogenously determined. However, we shall see that in an economy with infinitely many agents, the value of $n$ should, indeed, be infinitely large. Since, $\delta \lambda / \delta p_{i}=0$ and $v^{\prime}\left(c_{i}\right)=\lambda p_{i}$ from the constrained utility maximization by the agents, we may define the elasticity of demand for each good as $\varepsilon_{i}=-v^{\prime} / v^{\prime \prime} c_{i}{ }^{1}$ It is further assumed that

1 When $\delta \lambda / \delta p_{i} \neq 0$, the elasticity of demand $\varepsilon_{i}$ will be given by

$$
\varepsilon_{i}=-\frac{v^{\prime}}{v^{\prime \prime} c_{i}}\left(1+\frac{p_{i}}{\lambda}\left(\frac{\delta \lambda_{i}}{\delta p_{i}}\right)\right)
$$

where $\lambda$ denotes the marinal utility of income. Our definition $\varepsilon_{i}=-v^{\prime} / v^{\prime \prime} c$ holds when $\delta \lambda / \delta p_{i}=0$.

Originally published in Keio Economic Studies (1990), 26, 65-71. 


$$
d \varepsilon_{i} / d c_{i} \leqq 0 \quad \text { and } \quad \lim _{c_{i} \rightarrow 0} \varepsilon_{i}>1
$$

The profit maximizing price in the model is given by,

$$
p=(\varepsilon / \varepsilon-1) \beta w
$$

This together with the longrun condition for zero profits,

$$
p x-(\alpha+\beta x) w=0
$$

provides us with the equilibrium values for wage $(w)$, per capita consumption for each commodity $(c)$, size of each firm $(x)$ and the variety of outputs produced $(n)$. Using Eqs. (6) and (7), the equilibrium condition for a monopolistically competitive economy may be written as,

$$
\frac{\alpha}{\beta L c}+1=\frac{\varepsilon}{\varepsilon-1}
$$

Eq. (5) establishes the existence and the uniqueness of the equilibrium. One can easily show that in equilibrium,

$$
x=\alpha /(p / w-\beta) \quad \text { and } \quad n=L /(\alpha+\beta x)
$$

This is equilibrium under monopolistic competition, because price is greater than marginal cost $(\beta w)$ but the zero profit condition prevails. Note, the price is greater than the marginal cost because the size of the firm is finite. In a large economy where $L$ approaches infinity, if the size of the firms $(x)$ also approaches infinity, then the equilibrium under monopolistic competition converges to the competitive solution. On the other hand, if $x$ is finite in the limit, monopolistic competition continues to exist in the large economy.

\section{THE ECONOMY IN THE LARGE}

The existence of a limit for the size of firms when $L$ approaches $\infty$ is of crucial importance for the monopolistic competition to exist in a large economy. Before we discuss it, we need to prove proposition 1.

Proposition 1. As L approaches infinity, c tends to 0 and n approaches infinity.

Proof. By Eq. (5), $\varepsilon /(\varepsilon-1)$ and $c$ can not vary in opposite directions. Therefore, by Eq. (8), $\lim _{L \rightarrow \infty} c=0$. Again, by Eq. (9), $n=1 /(\alpha / L)+\beta c$ ). Hence, $\lim _{L \rightarrow \infty} n=1 / \beta\left(\lim _{L \rightarrow \infty} c\right)=\infty$.

(Q.E.D.)

Proposition 2. If $\lim _{c \rightarrow 0} \varepsilon=\bar{\varepsilon}, 1<\bar{\varepsilon}<\infty$, then monopolistic competition prevails in a large economy. On the other hand, if $\lim _{c \rightarrow 0} \varepsilon=\infty$, then the equilibrium under monopolistic competition converges to the competitive solution (i.e., price converges 
to the marginal cost.

Proof. Eq. (8) may be rewritten as, $\alpha / \beta x+1=1 /(1-(1 / \varepsilon))$. By our assumption (Eq. (5)), $\lim _{c \rightarrow 0} \varepsilon>1$. From proposition 1, it follows that if $\lim _{c \rightarrow 0} \varepsilon$ exists then $\lim _{L \rightarrow \infty} x$ is finite. On the other hand if $\lim _{c \rightarrow 0} \varepsilon$ does not exist then $\lim _{L \rightarrow \infty} x$ also does not exist., i.e., $x$ tends to $\infty$ as $L$ tends to $\infty$.

(Q.E.D.)

Next, we shall prove that if $\lim _{c \rightarrow 0} \varepsilon$ is finite and a monopolistically competitive economy does not converge to a competitive economy when $L$ tends to $\infty$, then the equilibrium under monopolistic competition satisfies the condition for pareto optimality in the limit. The following proposition states the condition for pareto optimality in the finite-agent economy.

Proposition 3. In the finite-agent economy, the condition for pareto optimality is given by the following equation.

$$
\frac{\alpha}{\beta L c}+1=\frac{v}{v^{\prime} c}
$$

Proof. For a fixed value of $n$, using Eqs. (1) and (2), we obtain the production possibility frontier for the economy as, $L=n \alpha+\sum_{i=1}^{n} x_{i}$. Apparently, the gradient of the production possibility frontier is the unit vector, $(1,1, \cdots, 1)$. Again, from Eq. (3), we observe that if $c_{i}=c$ for all $i$ and all agents, then the implicit price vector is also an unit vector. Therefore, the marginal rate of transformation equals the marginal rate of substitution: $M R T_{i j}=M R S_{i j}=1 ; i, j=i, 2, \cdots, n$. Moreover, the income of all the agents are same $(n c)$. Since the utility function is strictly concave and is of the form given by Eq. (3), at any allocation other than $c_{i}=c$ for all $i, M R S_{i j} \neq 1$ for all $i, j$. Hence, $l=M R T_{i j} \neq M R S_{i j}$ for all $i, j$. That is to say, the consumption bundle $(c, c, \cdots, c)$ is the only pareto optimal allocation in our economy for a given value of $n$. If $n$ is a control-variable, then pareto optimality requires $n$ to be chosen as follows:

Maximize $n v(c)$ subject to $n=L /(\alpha+\beta L C)$

The first order condition for the above optimization problem yields,

$$
\frac{\alpha}{\beta L c}+1=\frac{v}{v^{\prime} c}
$$

which determines the pareto optimal values for $c$ and $n$.

(Q.E.D.)

Comparing Eq. (8) with Eq. (10), it is obvious that in a finite agent economy the equilibrium under monopolistic competition, in general, is not pareto optimal. The equilibrium will satisfy conditions of pareto optimality if $v / v^{\prime} c=\varepsilon /(\varepsilon-1)$ which is not true in general. In proposition 2 , we established that if $\lim _{c \rightarrow 0} \varepsilon$ exists then monopolistic competition prevails in the large economy. Now, we shall prove that 
if $\lim _{c \rightarrow 0} \varepsilon$ exists, then the size of firms under monopolistic competition must converge to the optimal size. This is shown in the following way. From Eqs. (8) and (10), the size of the monopolistically competitive firms and the optimal size are given by $1 / \beta x=\varepsilon /(\varepsilon-1)$ and $1 / \beta x=v / v^{\prime} c$ respectively. In the following proposition we shall show that $\lim _{c \rightarrow 0}\left(v / v^{\prime} c\right)=\bar{\varepsilon} /(\bar{\varepsilon}-1)$, establishing the convergence of equilibrium under monopolistic competition to the pareto optimal allocation.

Proposition 4. If $\lim _{c \rightarrow 0} \varepsilon=\bar{\varepsilon}, 1<\bar{\varepsilon}<\infty$, then the size of the monopolistically competitive firms converges to the optimal size as the number of agents $(L)$ tends to infinity.

Proof. We are required to prove that $\lim _{c \rightarrow 0}\left(v / v^{\prime} c\right)=\bar{\varepsilon} /(\bar{\varepsilon}-1)$. Since, $v(c)$ is concave in $c$, we know that $v>v^{\prime} c$ for $c>0$. Therefore, $\lim _{c \rightarrow 0}\left(v / v^{\prime} c\right) \geqq 1$. Since $v(0)=0$, it is possible only if $\lim _{c \rightarrow 0} v^{\prime} c=0$. Now we know that $\lim _{c \rightarrow 0} v=0$ and $\lim _{c \rightarrow 0} v^{\prime} c=0$. By applying L'Hopital's rule we obtain,

$$
\lim _{c \rightarrow 0} \frac{v}{v^{\prime} c}=\lim _{c \rightarrow 0} \frac{v^{\prime}}{v^{\prime \prime} c+v^{\prime}}=\lim _{\varepsilon \rightarrow 0} \frac{\varepsilon}{\varepsilon-1}=\frac{\bar{\varepsilon}}{\bar{\varepsilon}-1}
$$

(Q.E.D.)

\section{THE CONCLUDING REMARKS}

Dixit and Stiglitz (1977) provided an example where the equilibrium under monopolistic competition was pareto optimal. The result followed from the assumption of a particular form of utility function: $U=U\left(c_{0},\left\{\sum x_{i}^{\beta}\right\}^{1 / \beta}\right)$ which was maximised by the representative individual subject to the budget constraint $c_{0}+p_{i} x_{i}=I$. In this case, the maximization procedure has two steps: (1) Maximize $U=\left(c_{0}, c_{1}\right)$ subject to $c_{0}+c_{1}=I$ and (ii) Maximize $\left(\sum x_{i}^{\beta}\right)^{1 / \beta}$ subject to $\sum p_{i} x_{i}=c_{1}$. The combination of constant-elasticity utility function and constant marginal utility of income produces the pareto optimality of monopolistic competition. The reader may easily check it by using $v(c)=c^{q}, 0<q<1$ and showing $\varepsilon /(\varepsilon-1)=v / v^{\prime} c=1 / q$. Note, although $\lim _{c \rightarrow 0} v^{\prime}$ and $\lim _{c \rightarrow 0} v^{\prime \prime} c$ do not exist in this case, one can show that $\lim _{n \rightarrow \infty}\left(\delta \lambda / \delta p_{i}\right)=0$ (see Appendix). We have not taken this route by specifying the form of $v(c)$. In our case, for a finite agent economy, monopolistic competition may not lead to pareto optimality. But if monopolistic competition prevails as the number of agents tends to infinity, the size of firms under monopolistic competition converges to the optimal size.

\section{APPENDIX}

Proposition A.1. If $\lim _{c \rightarrow 0} v^{\prime}$ and $\lim _{c \rightarrow 0} v^{\prime \prime} c$ exists (bounded in the limit) then $\lim _{n \rightarrow \infty}\left(\delta \lambda / \delta p_{i}\right)=0$ in the equilibrium. 
Proof. By the first order condition of a maximum $\lambda=v^{\prime}\left(c_{k}\right) / p_{k}, k=1,2, \cdots, n$. Differentiating with respect to $p_{i}$, we obtain:

$$
\frac{\delta \lambda}{\delta p_{i}}=\frac{v^{\prime \prime}}{p_{i}} \cdot \frac{\delta c_{i}}{\delta p_{i}}-\frac{v^{\prime}}{p_{i}^{2}}=\frac{v^{\prime \prime}}{p_{j}} \cdot \frac{\delta c_{j}}{\delta p_{i}} \quad(i \neq j)
$$

At equilibrium $\left(c_{j}=c_{i}, p_{j}=p_{i}\right)$, we get the following relationship:

$$
\frac{\delta c_{i}}{\delta p_{i}}=\frac{v^{\prime}}{v^{\prime \prime} p_{i}}+\frac{\delta c_{j}}{\delta p_{i}} \quad(i \neq j)
$$

Next, consider the budget constraint $\sum p_{i} c_{i}=w$. In equilibrium $p_{k}=p$ for all $k$ and we can normalize $(w, p)$ in such a way that $p_{k}=p=1$. We shall show that under normalization $\lim _{n \rightarrow \infty}\left(\delta \lambda j \delta p_{i}\right)=0$. If under normalized prices $\lambda$ is invariant with respect to price changes, then it is also invariant when the prices are not normalized. Under normalization differentiating the budget constraint with respect to $p_{i}$, we obtain:

$$
c_{i}+(n-1) \frac{\delta c_{j}}{\delta p_{i}}+\frac{\delta c_{i}}{\delta p_{i}}=0
$$

From Eqs. (a.2) and (a.3) we get:

$$
\frac{\delta c_{j}}{\delta p_{i}}=-\frac{1}{n}\left(c_{i}+\frac{v^{\prime}}{v^{\prime \prime} p_{i}}\right)
$$

Substituting the above in Eq. (3) we obtain:

$$
\frac{\delta \lambda}{\delta p_{i}}=\frac{1}{n p_{i}}\left\{-\left(v^{\prime \prime} c_{i}+\frac{v^{\prime}}{p_{i}}\right)\right\}
$$

Since, under normalization $p_{i}=1$ for all $i$,

$$
\frac{\delta \lambda}{\delta p_{i}}=\frac{1}{n}\left\{-\left(v^{\prime \prime} c_{i}+v^{\prime}\right)\right\}
$$

We know $n c_{i}=w$ since $p_{i}=1$ for all $i$. Therefore it is obvious from Eq. (a.4) that $\lim _{n \rightarrow \infty}\left(\delta \lambda / \delta p_{i}\right)=0$ if $\lim _{c_{i} \rightarrow 0} v^{\prime}\left(c_{i}\right)$ and $v^{\prime \prime}\left(c_{i}\right) c_{i}$ exist (bounded in the limit).

(Q.E.D.)

Remark 1. At equilibrium, the share of the $i$-th commodity in total expenditure is $1 / n$. When $n$ is infinitely large, the expediture on the $i$-th commodity is an insignificant part of the total budget. In this case a change in $p_{i}$ has insignificant effect on the marginal utility of income.

Remark 2. The conditions, $\lim _{c \rightarrow 0} v^{\prime}$ and $\lim _{c \rightarrow 0} v^{\prime \prime} c$ exist, are only sufficient conditions for $\lim _{n \rightarrow \infty}\left(\delta \lambda / \delta \mathrm{p}_{i}\right)=0$. An interesting case arises if $v=c^{q}, 0<q<1$. In 
this case both $\lim _{c \rightarrow 0} v^{\prime}$ and $\lim _{c \rightarrow 0} v^{\prime \prime} c$ do not exist but $\lim _{n \rightarrow \infty}\left(\delta \lambda / \delta p_{i}\right)=0$. For $v=c^{q}$, Eq. (a.4) yields,

$$
\frac{\delta \lambda}{\delta p_{i}}=-\frac{1}{n c_{i}} q^{2} c_{i}^{q}=-\frac{1}{w} q^{2} c_{i}^{q}
$$

Therefore, $\delta \lambda / \delta p_{i}$ tends to 0 as $n$ approaches infinity.

\section{University of Hull}

\section{REFERENCES}

Dixit, A. K. and J. E. Stiglitz, "Monopolistic Competition and Optimum Product Diversity", American Economic Review, 67 (1977), 297-308.

Dixit, A. K. and V. Norman, Theory of International Trade, James Nisbet \& Co: Herts, U.K., 1980.

Helpman, E., "Increasing Returns, Imperfect Markets and Trade Theory", in R. W. Jones and P. B. Kenen eds. Handbook of International Economics, North Holland: New York, 1984, 325-365.

Horn, H., "Product Diversity, Trade and Welfare", in H. Kierkowski ed. Monopolistic Competition and International Trade, Clarendon: Oxford, 1984, 51-68.

Krugman, P., "Increasing Returns, Monopolistic competition and International Trade, Journal of International Economics, 4 (1979), 469-479. 